\title{
La memoria espacial, y los niveles de BDNF en el hipocampo, disminuyen en ratas adolescentes deprimidas farmacológicamente con reserpina ${ }^{1}$
}

\author{
Paul Ruiz ${ }^{2}$, Aldo Calliari ${ }^{3}$, Patricia Genovese ${ }^{4}$, Ricardo Pautassi ${ }^{5}$ \\ Universidad de la República-Uruguay ${ }^{2,3,4}$, Instituto de Investigación Médica M. \\ y M. Ferreyra-Argentina ${ }^{2,5}$
}

\begin{abstract}
Los cuadros depresivos en humanos cursan con significativas alteraciones en la memoria espacial. Este trabajo estudió esta función en un modelo animal de depresión. Ratas Wistar $(\mathrm{n}=28)$, machos y hembras, fueron administradas con reserpina $(0.0$ o $1.0 \mathrm{mg} / \mathrm{kg} / \mathrm{cada} 2$ días por cuatro días). Luego del tratamiento se evaluó la función motora a través de la prueba de la barra y la respuesta de ansiedad a través de un laberinto elevado en cruz. Posteriormente, los animales fueron entrenados durante una semana (en sesiones diarias de 5 minutos) en un laberinto de múltiples T que indaga la memoria espacial. Luego se repitió la evaluación en el laberinto elevado en cruz y los animales fueron sacrificados para disecar el hipocampo y medir los niveles de BDNF a través de western blot. En la prueba de la barra los animales tratados con reserpina tuvieron una respuesta motora con igual agarre, pero menor movilidad, que los controles. En ambas tomas del laberinto elevado en cruz los animales tratados con reserpina pasaron significativamente menos tiempo en los brazos abiertos y exhibieron una latencia más elevada para entrar por primera vez en los brazos cerrados. Los animales tratados con reserpina tardaron significativamente más tiempo en resolver el laberinto de múltiples T que los controles, y cometieron más errores, efecto que estuvo asociado a niveles menores de BDNF en el hipocampo. Estos resultados sugieren que la reserpina indujo una
\end{abstract}

1 La investigación que da origen a los resultados presentados en esta publicación recibió fondos de la Agencia Nacional de Investigación e Innovación (ANII) bajo el código POS EXT_2014_1_105 877, y de la Comisión Sectorial de Investigación Científica de la Universidad de la República (CSIC-UdelaR). Agradecemos a los estudiantes Mario Lopez y Lucas Maidana (FVet-UdelaR) por la colaboración en la desgrabación de videos.

2 PhD en Psicología. Docente en Universidad de la República, Montevideo, Uruguay. Dirección postal: Universidad de la República. Lasplaces 1550, C.P 11600, Montevideo, Uruguay. Contacto: paulruiz@fvet.edu.uy. https://orcid.org/0000-0003-3180-9614

$3 \mathrm{PhD}$ en Fisiología. Docente Universidad de la República, Uruguay. Dirección postal: Universidad de la República. Lasplaces 1550, C.P 11600, Montevideo, Uruguay. Contacto: aldocalliari@gmail.com. https://orcid.org/0000-0002-3581-347X

4 MSc. Docente Universidad de la República, Uruguay. Dirección postal: Universidad de la República. Lasplaces 1550, C.P 11600, Montevideo, Uruguay. Contacto: patriciagenovese@ gmail.com. https://orcid.org/0000-0001-8876-2015

5 PhD en Biología. Docente Universidad Nacional de Córdoba, Argentina. Dirección postal: Instituto de Investigación Médica Mercedes y Martín Ferreyra (INIMEC-CONICET), Friuli 2434, Córdoba, C.P 5016, Argentina. Contacto: rpautassi@gmail.com. https://orcid. org/0000-0003-1876-2321 
depresión experimental, sin compromiso motor, que estuvo asociada a déficits en la adquisición de información espacial.

Palabras clave: Memoria espacial, BDNF, ratas, reserpina.

Spatial memory, and hippocampal BDNF levels, decrease in adolescent reserpine depressed rats.

Depression is associated with significant deficits in spatial memory. The present study assessed spatial memory in an animal model of depression. Wistar rats $(\mathrm{n}=28)$, males and females, were administered reserpine $(0.0$ or $1.0 \mathrm{mg} / \mathrm{kg} /$ every 2 days for 4 days $)$. At termination of the treatment the rats were tested for motor function (bar test) and for anxiety response (elevated plus maze). The animals were then trained for a week (daily sessions of 5 min) in a multiple T-maze, which assesses spatial memory. The elevated plus maze test was then repeated and subsequently the animals were sacrificed and the hippocampus dissected to measure BDNF levels through a Western blot. In the bar test, the reserpine-treated rats exhibited similar grasping, yet reduced mobility, when compared to vehicle-treated peers. Reserpine-treated rats exhibited, in both elevated plus maze tests and when compared to control counterparts, significantly less time spent in the open arms, and significantly greater latency to enter, for the first time, in the closed arms. They also spent significantly more time to complete the multiple T-maze and made more performance errors than controls in this test, a profile that was associated with lower levels of BDNF at the hippocampus. These results suggest that reserpine induced a depression-like phenotype, without gross motor alterations, which was associated with spatial memory deficits.

Keywords: Spatial memory, BDNF, rats, reserpine.

Memória espacial e níveis de BDNF no hipocampo diminuem em ratos adolescentes deprimidos farmacologicamente com reserpina.

Depressão em humanos apresenta depressão alteraçôes significativas na memória espacial. Este trabalho estudou essa função em um modelo animal de depressão. Ratos Wistar ( $\mathrm{n}=$ 28), machos e fêmeas, foram administrados com reserpina ( 0.0 ou $1.0 \mathrm{mg} / \mathrm{kg} /$ a cada 2 dias por quatro dias). Após o tratamento, a função motora foi avaliada através do teste de bar e da resposta de ansiedade através de um labirinto cruzado elevado. Posteriormente, os animais foram treinados por uma semana (em sessóes diárias de 5 minutos) em um labirinto multi-T que investiga a memória espacial. Na avaliação foi repetida no labirinto elevado em cruz e os animais foram sacrificados para dissecar o hipocampo e medir os níveis de BDNF através de western blot. No teste de barra, os animais tratados com reserpina tiveram uma resposta motora com a mesma aderência, mas menor mobilidade, que os controles. Em ambas as entradas do labirinto elevado na cruz, os animais tratados com reserpina gastaram significativamente menos tempo nos braços abertos e exibiram uma maior latência para entrar nos braços fechados pela primeira vez. Os animais tratados com reserpina levaram significativamente mais tempo para resolver o labirinto multi- $T$ do que os controles, e cometeram mais erros, um efeito que foi associado com níveis mais baixos de BDNF no hipocampo. Esses resultados sugerem que a reserpina induziu uma depressáo experimental, sem comprometimento motor, associada a déficits na aquisição de informaçóes espaciais.

Palavras-chave: Memória espacial, BDNF, ratos, reserpina. 
La memoria espacial y los niveles de BDNF en el hipocampo / Ruiz et al.

Mémoire spatiale et niveaux de BDNF dans l'hippocampe, diminution chez les rats adolescents déprimés pharmacologiquement avec la réserpine

Dépression chez l'homme présentent des altérations importantes de la mémoire spatiale. Ce travail a étudié cette fonction dans un modèle animal de dépression. Des rats Wistar ( $\mathrm{n}$ = 28), mâles et femelles, ont été administrés avec de la réserpine $(0.0$ ou $1.0 \mathrm{mg} / \mathrm{kg} /$ tous les 2 jours pendant quatre jours). Après le traitement, la fonction motrice a été évaluée par le test des barres et la réponse anxieuse par un labyrinthe croisé surélevé. Par la suite, les animaux ont été entraînés pendant une semaine (par sessions quotidiennes de 5 minutes) dans un labyrinthe multi-T qui étudie la mémoire spatiale. Ensuite, l'évaluation a été répétée dans le labyrinthe en croix et les animaux ont été sacrifiés pour disséquer l'hippocampe et mesurer les taux de BDNF par Western blot. Lors du test des barres, les animaux traités à la réserpine présentaient une réponse motrice avec la même adhérence, mais avec une mobilité moindre, que les témoins. Dans les deux prises du labyrinthe surélevé dans la croix, les animaux traités à la réserpine ont passé beaucoup moins de temps dans les bras ouverts et ont montré une plus grande latence pour entrer dans les bras fermés pour la première fois. Les animaux traités avec de la réserpine ont mis beaucoup plus de temps à résoudre le labyrinthe multi-T que les témoins et ont commis plus d'erreurs, un effet associé à des niveaux inférieurs de BDNF dans l'hippocampe. Ces résultats suggèrent que la réserpine a induit une dépression expérimentale, sans compromis moteur, associée à des déficits dans l'acquisition d'informations spatiales.

Mots clés: mémoire spatiale, BDNF, rat, réserpine. 
El estado anímico refleja el momento afectivo y emocional de un individuo, siendo más duradero y menos específico que la respuesta a una situación concreta. Cuando es anormalmente bajo se llama depresión y cuando es anormalmente alto se llama manía, existiendo otros posibles estados como la ciclotimia y la bipolaridad. Estos cuadros son agrupados y llamados Trastornos de estado de ánimo (CIE-10-OMS) siendo de particular interés dada la prevalencia en la población humana y los inconvenientes que acarrea para la salud, el bienestar y el desarrollo de los individuos. Dentro de estos trastornos la depresión es el de mayor prevalencia, además de ser la enfermedad crónica que más compromete la salud (Moussavi et al., 2007). Los resultados sobre la carga mundial de las enfermedades (Global Burden of Disease) efectuado por la Organización Mundial de la Salud demostraron que la depresión unipolar es la principal causa de años de vida perdidos por discapacidad (Gómez-Restrepo et al., 2004).

Diferentes funciones cognitivas (e.g. atención, memoria, funciones ejecutivas y toma de decisiones; Chamberlain \& Sahakian, 2004, 2006; Goodale, 2007) estan alteradas en humanos afectados por dichos trastornos, y también en modelos preclínicos. Los modelos animales de depresión no recrean todo el espectro de la patología y han recibido críticas relevantes (Leite \& Perez-Acosta, 2001), pero son utiles para poder determinar etiopatogenia (Escorihuela, Fernandez-Terual, 1998; Overmier, 2007; Laborda, 2009), así como consecuencias y eventuales tratamientos (Deussing, 2006; Healy, 1987; Nestler \& Hyman, 2010). Específicamente hablando de la memoria, y dentro de ella de la memoria espacial, numerosos reportes muestran que en humanos y animales deprimidos esta función se encuentra alterada, e incluso sugieren que memoria espacial y estado de ánimo compartirían el mismo sustrato neurobiológico (Held et al., 2007; Sun \& Alkon, 2005).

En lo que respecta a la neurobiología de estos trastornos, uno de los aspectos que se ha estudiado más es qué tipos de neurotransmisores 
se encuentran alterados. La dopamina se ha visto implicada en la depresión y en la manía, viéndose disminuída en la primera y aumentada en la segunda (Diehl \& Gershon, 1992; Ruiz, Calliari, Genovese et al., 2018). Junto con la teoría dopaminérgica sobre la etiología de los trastornos de estado de ánimo, se ha también involucrado al factor neurotrópico derivado del cerebro (BDNF, por sus siglas en inglés). La variación de dicho factor podría tener un papel relevante en el desarrollo de estos trastornos, viéndose alterado en estructuras como el hipocampo, responsable del almacenamiento de información y aprendizaje (Armas et al., 2010; Duncan et al., 2009; Frey et al., 2006; Jiang et al., 2005).

La depresión se puede modelar en animales de laboratorio de varias formas, a través de la inducción de estrés crónico, físico o social (Krishnan \& Nestler, 2011), empleando de cepas de roedores criadas selectivamente por expresar o no rasgos asociados a la depresión (Overstreet et al., 2007), o mediante la administración de fármacos que disminuyen los niveles de monoaminas en el sistema nervioso central, como la reserpina (RES; Czeh et al., 2016; Ikram \& Haleem, 2017; Khadrawy et al. , 2017; Ruiz, Genovese et al., 2018). Esta droga ha demostrado ser útil para generar comportamientos depresivo en ratas reproduciendo muchas de las variables conductuales y neuroendócrinas vistas en humanos deprimidos. Por ejemplo, en nuestro laboratorio hemos recientemente observado que ratas tratadas crónicamente con RES expresaban actividad reducida en la prueba de campo abierto, menor actividad motora y respuesta ansiogénica en el laberinto elevado en cruz y la caja de luz/oscuridad, menor consumo de solución azucarada, niveles de dopamina insular disminuidos, así como mayor consumo de alcohol que ratas tratadas con vehículo (Ruiz et al., 2017; Ruiz, Genovese et al., 2018).

El objetivo de este trabajo fue estudiar como se ve afectada la memoria espacial en un modelo farmacológico de depresión a través de la administración de RES. y analizar si este modelo animal reproduce lo visto en humanos con este trastorno. Para esto tratamos un grupo de ratas, hembras y macho, con reserpina y estudiamos como 
afecta la actividad motora, la respuesta ansiosa, la memoria espacial y los niveles de BDNF en el hipocampo.

El tratamiento y las evaluaciones se realizaron en el período 30-40 días postnatales, el cual representa el periodo de la adolescencia en la rata (Spear, 2000), momento de importante flexibilidad neurocognitiva (Hauser et al., 2015). La importancia de estudiar la depresión durante la adolescencia es que, de manera similar a los trastornos de ansiedad, esta patología usualmente surge durante la adolescencia (McGorry et al., 2011). En el presente trabajo, asimismo, emplearemos hembras y macho con el objetivo de describir potenciales dimorfismos sexuales en los fenómenos bajo análisis. Las mujeres son más propensas a ser diagnosticadas con depresión, particularmente luego del inicio de la pubertad (Rocha et al., 2013).

\section{Método}

\section{Diseño experimental y procedimientos}

Se empleó un diseño factorial 2 (tratamiento farmacológico: RES, vehículo) x 2 (sexo: macho, hembra), con 7 sujetos en cada uno de los 4 grupos. La Figura 1 presenta un esquema del procedimiento experimental empleado. Más en detalle, el procedimiento implicó la administración de RES $(1.0 \mathrm{mg} / \mathrm{kg} / \mathrm{cada} 48$ horas, IP, por un total de 4 administraciones) o vehículo (VEH) durante los días postnatales (DP) 30 a 36. Luego del tratamiento se evaluó la función actividad motora a través de la prueba de la barra (DP 37) y, 24 horas más tarde, la respuesta de ansiedad mediante el laberinto elevado en cruz. Posteriormente, los animales fueron entrenados durante 7 días (en sesiones diarias de 5 minutos, DPs 39 a 45) en un laberinto de múltiples T. La evaluación en el laberinto elevado en cruz fue repetida el DP 46 y el DP 47 los animales fueron sacrificados y se disecó el hipocampo para medir los niveles de BDNF a través de western blot. 


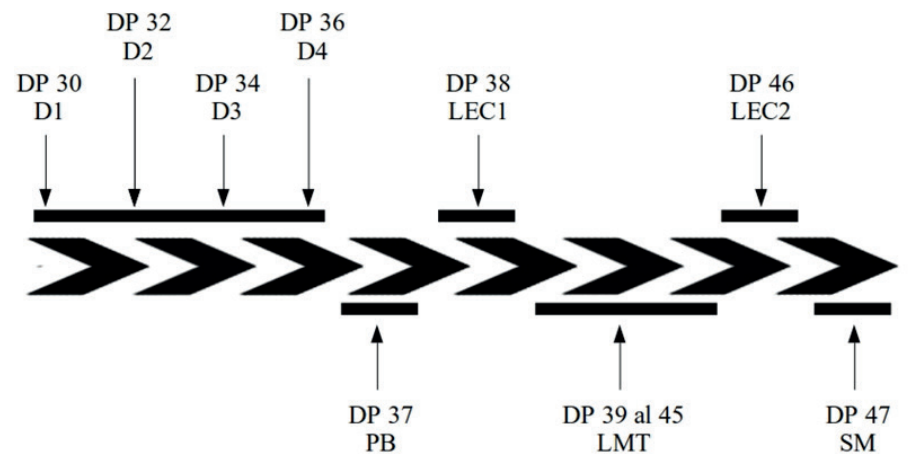

$\mathrm{DP}=$ Día postnatal $/ \mathrm{D}=$ Dosis $/ \mathrm{PB}=$ Prueba de la barra $/ \mathrm{LEC}=$ Laberinto elevado en cruz LMT $=$ Laberinto en múltiple $\mathrm{T} / \mathrm{SM}=$ Sacrificio y muestreo

Figura 1. Secuencia temporal de procedimientos experimentales realizados para la evaluación del modelo de depresión inducido por la administración de reserpina, desde el día postnatal (DP) 30, donde se inició el experimento, al día postnatal 47 donde los animales fueron sacrificados. La dosis administrada fue de 0.0 o $1.0 \mathrm{mg} / \mathrm{k}$ de reserpina, en los DPs 30, 32, 34, y 36. Posteriormente los animales fueron evaluados en la prueba de la barra, en el laberinto elevado en cruz (en dos ocasiones) y en el laberinto de múltiples $\mathrm{T}$.

\section{Animales}

Se empleó un total de 28 ratas Wistar adolescentes (14 machos, 14 hembras), criadas en la Unidad de Reactivos Biológicos de la Facultad de Medicina de la Universidad de la República (URBE-Fmed-UdelaR). Se hospedaron en cajas separadas por sexo en un cuarto con temperatura, humedad y luz controlada (12 horas luz, $21 \pm 1{ }^{\circ} \mathrm{C}, 80 \%$ ). La reserpina (Sigma-Aldrich, USA) fue vehiculizada en solución fisiológica y administrada de manera intraperitoneal a un volumen de $0.01 \mathrm{ml}$ por gramo de peso corporal $(0,01 \mathrm{ml} / \mathrm{g}$ ) (Ruiz et al., 2017). El protocolo experimental fue aprobado por la Comisión de Ética en Uso de Animales de la Facultad de Veterinaria de UdelaR [(CEUAFVet-UdelaR) Exp n¹11130-000658-13]. 


\section{Pruebas de comportamiento aplicadas}

Prueba de la barra

La prueba fue aplicada el DP 37 con el objetivo de estudiar como RES afectaba la actividad motora. Se empleó una barra de madera de $2.5 \mathrm{~cm}$ de diámetro a $70 \mathrm{~cm}$ del piso. Los animales se colocaron sobre la barra durante 1 minuto y fueron evaluados por dos personas entrenadas, con la siguiente escala (adaptado de Rosso et al., 2010); (1) movilidad normal y buen agarre; (2) reducida movilidad y buen agarre; (3) reducida movilidad y agarre medio; (4) ninguna movilidad y el agarre débil; (5) alteración de pie, sin agarre. Las sesiones se realizaron en la tarde, en una habitación iluminada con luz artificial blanca y aislada acústicamente.

\section{Laberinto elevado en cruz (LEC)}

El propósito fue confirmar la acción depresiva de la reserpina a nivel comportamental. El test de LEC es ampliamente usado para medir respuesta ansiosa (tiempo en brazos) y motora (entradas a brazos), la cual está asociada a la patología depresiva.

El aparato era una plataforma en forma de cruz, elevada a $50 \mathrm{~cm}$ del suelo, con dos brazos abiertos de 50x10 cm y dos cerrados de 50x10 con paredes de $40 \mathrm{~cm}$ de altura y descubierta en la parte superior. En cada sesión los animales se pusieron de manera individual en el centro del laberinto, enfrentando un brazo abierto. Los animales se retiraron del laberinto después de 5 minutos de exploración libre, limpiándose el laberinto entre animales. Las sesiones se realizaron en la tarde en una habitación iluminada con luz artificial blanca y aislada acústicamente. Las pruebas se grabaron por medio de una cámara de video ubicada en el techo de la habitación. Posteriormente, los archivos de video fueron evaluados, registrándose los siguientes patrones comportamentales: a) Frecuencia de entradas a brazos abiertos y cerrados; b) tiempo en cada uno de los brazos y en el centro; c) latencia para ingresar a brazos cerrados.

El laberinto fue aplicado dos veces con el objetivo de evaluar la continuidad del efecto de la reserpina a través de los días teniendo en cuenta el efecto de habituación a la prueba (Carobrez \& Bertoglio, 2005). 


\section{Laberinto en múltiple $T$}

Con el objetivo de investigar memoria espacial se aplicó un entrenamiento y posterior evaluación en un laberinto en múltiple T. Este es un test que desafía las habilidades de navegación y memoria espacial, consta de tres pasajes en forma de $\mathrm{T}$ unidas por un extremo, quedando un brazo como callejón y otro abierto donde el animal puede pasar a la próxima $T$ para salir del laberinto. La caja meta del laberinto esta equipada con comida (100 g aproximadamente. Los animales fueron expuestos a 16 horas de ayuno de comida antes de cada sesión para motivar la perfomance, luego de cada sesión fueron dejados en cajas con comida ad libitum hasta el inicio del ayuno registrándose el peso. Cada sesión duró 5 minutos, se realizó una vez por día durante siete días (DPs 39 al 45) en la tarde y comenzó con la introducción del animal en la primera de las T. La prueba se filmó y luego se registraron el número de errores de ejecución (i.e., cuando el animal se equivoca de brazo, asumiendo como correcto el camino más directo hacia la salida, o retrocede sobre sus pasos y vuelve a un brazo ya visitado) y el tiempo de ejecución (i.e., se considera que un ensayo ha finalizado cuando el animal tiene contacto con la comida) (Voicu \& Schmajuk, 2002). El laberinto fue limpiado luego del pasaje de cada animal.

\section{Análisis molecular por western blot}

El factor neurotrófico BDNF se midió en el hipocampo de 12 ratas seleccionadas aleatoriamente, en igual proporción de tratamientos y sexos, a través de western blot a partir de geles de poliacrilamida. Los animales fueron decapitados y se obtuvo los encéfalos posnecropsia para aislar el hipocampo. Este se homogeneizó y sus muestras fueron corridas en geles de poliacrilamida. Todas las electroforesis (SDSPAGE) fueron realizadas en minigeles de acrilamida-bis acrilamida y transferidos eléctricamente a un soporte de nitrocelulosa $(0.22 \mu \mathrm{m}$, Bio$\mathrm{Rad}$ ) por medio de la aplicación de $300 \mathrm{~mA}$ durante 75 minutos en un medio conteniendo Tris-KCl $25 \mathrm{mM}$, glicina $192 \mathrm{mM}$ y metanol $10 \% \mathrm{v} / \mathrm{v}$ (Towbin et al., 1979). Las membranas fueron incubadas con 
anticuerpo anti-BDNF (Santa Cruz Biotechnology), el sistema de detección utilizado fue de quimioluminiscencia para ser registrado sobre placas radiográficas. Las bandas de expresión obtenidas fueron cuantificadas por densitometría con el programa ImageJ (NIH).

\section{Análisis de datos}

Para el análisis de datos se usó el software Statistica $8.0^{\circ}$. En una primera instancia se realizaron ANOVA factoriales considerando como factores independientes, entre grupos, a Sexo y Tratamiento. Estos ANOVA indicaron la ausencia de efectos significativos de Sexo o de interacciones significativas Sexo x Tratamiento. Así entonces, los datos fueron colapsados por sexo, y las variables registradas en el laberinto elevado en cruz y en la densitometría de bandas del western blot fueron analizadas de forma independiente mediante prueba $t$ de Student. Los datos obtenidos en las las 7 sesiones del laberinto en múltiple $\mathrm{T}$ se analizaron mediante ANOVA de medidas repetidas (factor entre grupos: Tratamiento; factor de medidas repetidas: sesión). Los efectos significativos principales y las interacciones significativas fueron exploradas mediante la prueba post hoc de Fisher. Las variables categóricas registradas en la prueba de la barra fueron analizadas mediante $X^{2}$. El alfa fue de 0.05 y los resultados se expresan como media \pm SEM.

\section{Resultados}

Prueba de la barra y peso.

Las calificaciones observadas fueron la categoría 1 (movilidad normal y buen agarre) y la 2 (reducida movilidad y buen agarre), no obteniéndose ningún registro de las otras categorías. Se realizó un análisis de proporciones mediante $X^{2}$ para determinar si los grupos, tratados y no tratados diferían significativamente en la asignación de estas categorías. Los tratados con reserpina tuvieron más valores 2 (93\%), mientras los controles tuvieron significativamente más valores 1 (64\%) y la asociación entre valor y grupo fue significativa $\left(X^{2}=9.96, p<0.05\right)$ lo que 
evidencian un efecto de la reserpina en la movilidad, pero sin afectar el agarre. Observaciones informales indicaron que ninguno de los animales se cayó de la barra y que todos, los tratados con reserpina y los controles, exhibían un equilibrio adecuado. Estos resultados son coherentes con trabajos previos donde la actividad motora de animales tratados con reserpina se vio disminuida en un campo abierto (Ruiz et al., 2017), y en el laberinto elevado en cruz como se explica a continuación.

No se encontraron diferencias significativas en el peso de los grupos, ni al finalizar el tratamiento ni en al momento del sacrificio; lo que evidencia que la reserpina no afecto el desarrollo de los animales.

\section{Tabla 1}

Peso, prueba de la barra y laberinto elevando en cruz (LEC)

\begin{tabular}{|c|c|c|c|c|c|c|}
\hline & & \multicolumn{2}{|c|}{ Reserpina } & \multicolumn{2}{|c|}{ Control } & \multirow[b]{2}{*}{$t$} \\
\hline & & M & E.E & M & E.E & \\
\hline \multirow{7}{*}{ LEC toma 1} & Peso al finalizar tratamiento & 114.2 & 22.6 & 111.1 & 23.5 & ns \\
\hline & Latencia para ingresar a brazos cerrados (segs) & 24.6 & 22.4 & $6.5^{*}$ & 3.9 & $2.86^{*}$ \\
\hline & Tiempo en brazos abiertos (segs) & 4.6 & 9.5 & $28.1^{*}$ & 32.4 & $2.6^{*}$ \\
\hline & Tiempo en brazos cerrados (segs) & 260.4 & 28.9 & 254.3 & 21.1 & ns \\
\hline & Tiempo en el centro (segs) & 14.5 & 23.3 & 14.8 & 15.15 & ns \\
\hline & Frecuencia de entradas en brazos cerrados & 3.6 & 2.3 & $6.3^{*}$ & 3.1 & $2.63^{*}$ \\
\hline & Frecuencia de entradas en brazos abiertos & 0.5 & 0.7 & $2.1^{*}$ & 1.6 & $3.45^{*}$ \\
\hline \multirow{7}{*}{ LEC toma 2} & Latencia para ingresar a brazos cerrados (segs) & 7.7 & 5.9 & $3.0^{*}$ & 1.7 & $2.9^{*}$ \\
\hline & Tiempo en brazos abiertos (segs) & 50.5 & 26.7 & 57.6 & 26.7 & ns \\
\hline & Tiempo en brazos cerrados (segs) & 158.1 & 41 & 145.1 & 30.4 & ns \\
\hline & Tiempo en el centro (segs) & 69.6 & 29.3 & 86.4 & 32.5 & ns \\
\hline & Frecuencia de entradas en brazos cerrados & 7.2 & 2.7 & $9.1^{*}$ & 2.3 & $2.08^{*}$ \\
\hline & Frecuencia de entradas en brazos abiertos & 4.8 & 2.1 & 6.0 & 4.1 & ns \\
\hline & Peso al sacrificio & 164.1 & 31.5 & 154.3 & 38.5 & ns \\
\hline
\end{tabular}

${ }^{*} p<0.05$

Nota: Medias y errores standard para pesos corporales y variables registradas en el laberinto elevado en cruz (LEC) y en la prueba de la barra, en animales tratados con reserpina y controles. Los datos se presentan colapsados por sexo (macho, hembra). Análisis preliminares indicaron la ausencia de efectos significativos de sexo, o de interacciones significativas sexo x tratamiento, sobre las variables registradas en el LEC y en la prueba de la barra. 


\section{Laberinto elevado en cruz}

En la primera toma los animales tratados con reserpina tardaron significativamente más tiempo en entrar a los brazos cerrados, permanecieron significativamente menos tiempo en los brazos abiertos, y exhibieron significativamente menos entradas a los brazos cerrados y abiertos. Mientras que en la segunda toma se mantuvo la diferencia entre grupos en la latencia para ingresar a los brazos cerrados, y en los ingresos a los mismos brazos. La descripción de todas estas variables se encuentra en la tabla 1 .

Estos resultados evidencian una ralentización en la actividad motora y una alteración en la respuesta a situaciones de riesgo, producto de la administración de reserpina. Estos resultados mantienen coherencia con los encontrados en trabajos previos en el campo abierto y en la prueba de la caja de luz oscuridad (Ruiz et al., 2017).

\section{Laberinto en múltiple $T$}

Los ANOVA arrojaron efectos principales significativos de sesión y de tratamiento, sobre el tiempo $\left[F_{(6,138)}=26.64, p<0.05\right.$ y $F_{(1,23)}=8.41$, $p<0.05)$, respectivamente] y sobre los errores de ejecución $\left[F_{(1,15)}=7.69\right.$, $p<0.05$ y $F_{(6,138)}=9.27, p<0.05$, respectivamente]. Los análisis post-hoc indicaron que la latencia y el tiempo de ejecución fueron bajando progresiva y significativamente desde la primera a la tercera sesión, para posteriormente elevarse significativamente en las sesiones $4 \mathrm{y}$ 6, las cuales no difirieron entre sí (datos descriptivos no mostrados). Más importante para los objetivos de nuestro trabajo, y como se grafica en la Figura 2, las ratas tratadas con reserpina tardaron significativamente más tiempo en resolver el laberinto $\left[F_{(1,25)}=8.94, p<0.05\right]$ y cometieron más errores $\left[F_{(1,25)}=6.94, p<0.05\right]$ que los controles en el promedio general de las sesiones. 

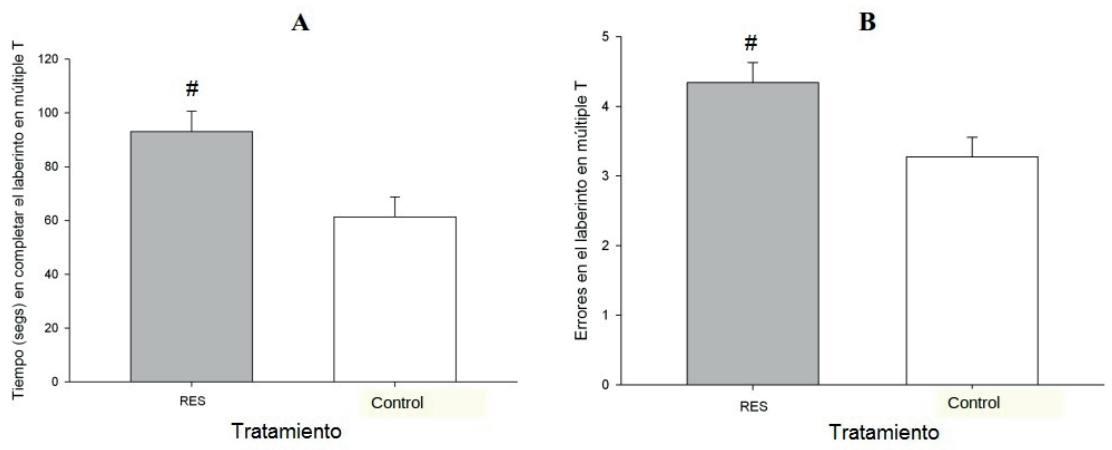

Figura 2. Tiempo (segundos) (A), y errores cometidos (B), para completar el laberinto en múltiple $T$ en animales tratados crónicamente con reserpina (RES) y en controles tratados con vehículo.. Los animales dosificados con reserpina exhibieron significativamente más tiempo y más errores para completar la prueba, en comparación con el grupo control. Los signos "\#” denotan estas diferencias significativas. Los datos se presentan colapsados por sexo (macho, hembra) y por sesión de entrenamiento. Análisis preliminares indicaron la ausencia de efectos significativos de sexo, o de interacciones significativas sexo $\mathrm{x}$ tratamiento, sobre las variables registradas en el LEC y en la prueba de la barra. Por favor referirse al texto para un detalle de los efectos significativos encontrados. Los valores expresan las medias \pm error estándar del promedio entre las sesiones.

\section{Niveles de BDNF en el hipocampo}

El análisis de la densidad de las bandas (i.e, cociente BDNF/ actina) mostró una tendencia a la disminución del BDNF en animales tratados con reserpina, la cual no alcanzo significancia estadística [ $t=-$ $1.76, p=0.1]$. 
A

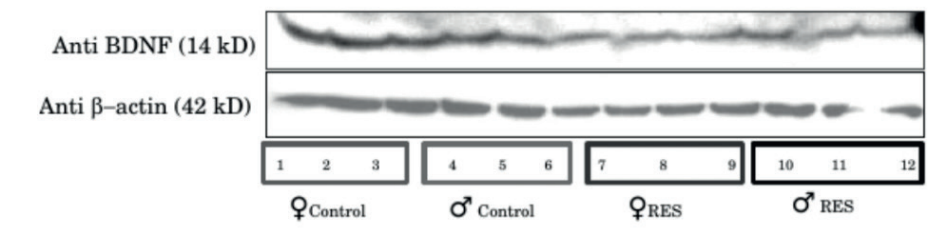

B

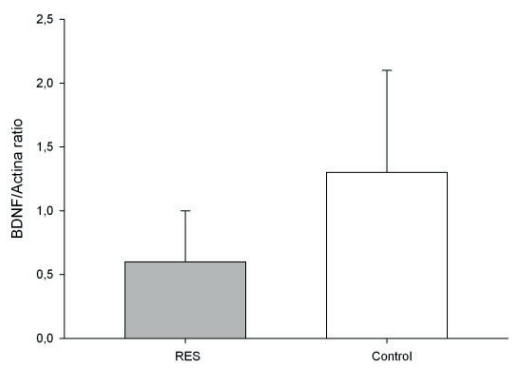

Figura 3.. Datos recogidos mediante Western blot de hipocampos, en ratas tratadas con reserpina o en controles tratados con vehículo (A). El análisis de la densidad de las bandas (BDNF/actina ratio, panel B) mostró una tendencia no significativa a la disminución del BDNF en animales tratados con reserpina, sin verse diferencias entre sexos. Los datos se presentan colapsados por sexo (macho, hembra). Análisis preliminares indicaron la ausencia de efectos significativos de sexo, o de interacciones significativas sexo x tratamiento. Los valores expresan las medias \pm error estándar del $\mathrm{BDNF} /$ actina ratio (B).

\section{Discusión}

El resultado principal de este trabajo es que la inducción experimental de depresión en ratas adolescentes induce alteraciones en el almacenamiento de la memoria espacial, que se correlaciona con alteraciones no significativas del factor de crecimiento BDNF en hipocampo. La efectividad de RES para inducir depresión fue confirmada mediante las mediciones realizadas en el LEC, las cuales indicaron que las ratas tratadas con RES exhibían conductas indicativas de ansiedad 
(e.g. menos tiempo pasado en los brazos abiertos) y de depresión dada la disminución de cruces entre brazos. Comparando las dos tomas del LEC se observo que en la segunda no se ven diferencias significativas entre grupos en las entradas y permanencia en los brazos cerrados, esto se puede explicar por la disminución en el tiempo del efecto de RES o por la habitación a la prueba como muestran otros estudios que ven este efecto en las mismas variables que nosotros (Carobrez \& Bertoglio, 2005).

La prueba de la barra, asimismo, indicó un enlentecimiento motor sin compromiso de fuerza muscular. Basado en la literatura epidemiológica, podía esperarse una exacerbación de estos efectos de RES, que sugieren alteraciones emocionales o hedónicas compatibles con un modelo de depresión experimental, en las hembras. Sin embargo, el perfil de resultados fue similar en machos y en hembras. A continuación discutiremos en detalle cada uno de estos resultados y sus implicancias.

El laberinto en múltiple $T$ es una prueba clásica, análoga al Water Morris Maze, para investigar la adquisición de memoria espacial en roedores (Voicu \& Schmajuk, 2002). La administración de RES altero la perfomance en esta prueba, aumentando el tiempo para completarla y, lo que es más importante, la cantidad de errores cometidos ante de llegar a la meta, resultados análogos a los encontrados en humanos con diagnóstico de depresión (Cunha et al., 2010; Erickson et al., 2012).

Podría sugerirse, como hipótesis alternativa, que el deterioro en la memoria espacial de los animales tratados con RES obedece a problemas musculares o a una alteración en el desarrollo inducida por la droga, en lugar de a un proceso depresivo (i.e., emocional o afectivo) concomitante. Sin embargo, no se observaron diferencias de peso ni diferencias en el agarre (i.e., en la prueba de la barra) que sugieran alteraciones graves del desarrollo o de la masa o fuerza muscular. Tampoco se observaron problemas de coordinación: los animales tratados con RES podían caminar en la barra, exhibiendo un equilibrio normal (Deacon, 2013). Más bien, los resultados sugieren que RES generó un enlentecimiento motor, probablemente resultado de alteraciones hedónica-emocionales, característico de los estados depresivos. Este 
enlentecimiento motor es consistente con resultados previos en los que se observó que ratas tratadas con RES exhibían menor distancia recorrida en la prueba de campo abierto (Ruiz et al., 2017), y también con los resultados obtenidos en el LEC, en el presente estudio. En este último laberinto la reserpina indujo conductas indicativas de un estado ansiogénico (menor tiempo pasado en brazos abiertos, en comparación con controles) y también de elentecimiento motor (mayor latencia a entrar por primera vez en los brazos cerrados).

Existe bibliografía que liga alteraciones en funciones cognitiva, y particularmente en memoria espacial, con los niveles hipocampales de BDNF. en el hipocampo (Egan et al., 2003). Esta última estructura esta especializada en el almacenamiento de información espacial. La disminución de BDNF hipocampal esta asociada, tanto en humanos como en animales, a déficits en el almacenamiento de la memoria espacial (Erickson et al., 2012; Mizuno et al., 2000). A su vez, BDNF está asociado con la neurogenesis en el hipocampo, y esta última se ha visto alterada en la depresión y se ha convertido en un objetivo terapéutico para los nuevos antidepresivos (Sahay \& Hen, 2007). Los niveles de monoaminas hipocampales también están asociados con la memoria espacial y los antidepresivos que mejoran los niveles de BDNF y serotonina hipocampales mejoran el almacenamiento de información espacial (Kiss et al., 2012). Esta asociación entre monoaminas y BDNF es coherente con resultados previos donde la reserpina bajaba los niveles de dopamina en la corteza insular (Ruiz et al., 2017, 2018). Basados en estos antecedentes, nuestra expectativa era que las alteraciones de memoria espacial inducidas por RES se asociaran con una menor expresión de BDNF hipocampal. Los resultados observados estuvieron en esa dirección, pero las diferencias observadas estuvieron lejos de ser estadísticamente significativas. Una posibilidad es que el diseño experimental no haya sido lo suficientemente poderoso, en términos de tamańo de muestra o administración de RES, para detectar efectos significativos. Esto es ciertamente posible, sin embargo debe notarse que los datos fueron colapsados por sexo, lo que aumentó la potencia estadística de los análisis realizados. 
Por último, es importante resaltar que en las variables estudiadas no se vio efecto del sexo. Existen trabajos que muestran que en humanos las mujeres son más propensas a la depresión (Rocha et al., 2013), y que en ensayos de navegación hombres y mujeres deprimidos tienen desempeños diferentes (Cornwell et al., 2010), además de haberse encontrado una disminución del volumen hipocampal en mujeres con historial de depresión (Sheline et al., 1999). En este trabajo no se encontraron diferencias, viendo que para las variables estudiadas RES no tiene efecto diferencial por sexos.

Los resultados obtenidos deben considerarse en el marco de importantes limitaciones. Solamente empleamos una dosis de RES, lo que limita la generalidad de los resultados obtenidos. Empleamos, asimismo, un diseño intrasujeto, en el cual los mismos animales fueron evaluados en diferentes tests, lo que abre la posibilidad que al menos parte de los resultados estén afectados por efectos interactivos entre tests. Finalmente, la droga utilizada (RES) es relativamente promiscua farmacológicamente, depletando los niveles centrales de todas las monoaminas, como demostramos en Ruiz et al. (2017, 2018). Los estudios futuros deberían emplear drogas que afecten de manera específica uno u otro sistema de neurotransmisión, como es el caso de DL-P-chlorophenylalanina (PCPA), un inhibidor selectivo de la síntesis de serotonina (Vitalis et al., 2007).

Más allá de estas limitaciones, los resultados obtenidos muestran similitudes entre los efectos de la reserpina en nuestro modelo animal y las alteraciones cognitivas vistas en humanos deprimidos. El tratamiento con reserpina generó consecuencias a nivel anímico (laberinto elevado en cruz) y cognitivo (laberinto de múltiple T), tanto en machos como en hembras, mostrando ser un modelo potencialmente útil para investigar esta problemática que afecta a humanos y evaluar potenciales tratamientos que inhiban esta condición. 


\section{Referencias}

Armas, G., Pezoa, E., Vasquez, J. (2010). Factor neurotrófico derivado del cerebro en el trastorno depresivo mayor. El Residente, 5(1), 19.21 .

Chamberlain, S., Sahakian, B. (2004). The neuropsychology of mood disorders. Current Psychiatry Reports, 6, 451-458. https://doi. org/10.1007/s11920-006-0051-x

Chamberlain, S., Sahakian, B. (2006). The neuropsychology of mood disorders. Current Psychiatry Reports, 8(6), 458-463. https://doi. org/10.1007/s11920-006-0051-x

Carobrez, A., Bertoglio, L. (2005). Ethological and temporal analyses of anxiety-like behavior: the elevated plus-maze model 20 years on. Neuroscience \& Biobehavioral Reviews, 29(8), 1193-205. https://doi.org/10.1016/j.neubiorev.2005.04.017

Cornwell, B. R., Salvadore, G., Colon-Rosario, V., Latov, D. R., Holroyd, T., Carver, F. W., ... Grillon, C. (2010). Abnormal hippocampal functioning and impaired spatial navigation in depressed individuals: evidence from wholehead magnetoencephalography. The American Journal of Psychiatry, 167(7), 836-844. https://doi.org/10.1176/appi. ajp.2009.09050614

Cunha, C., Brambilla, R., Thomas, K. (2010). A Simple role for BDNF in learning and memory? Frontiers in Molecular Neuroscience, 3, 1. https://doi.org/10.3389/neuro.02.001.2010

Czeh, B., Fuchs, E., Wiborg, O., Simon, M. (2016). Animal models of major depression and their clinical implications. Progress in Neuro-psychopharmacology \& Biological Psychiatry, 64, 293-310. https://doi.org/10.1016/j.pnpbp.2015.04.004

Deacon, R. (2013). Measuring motor coordination in mice. Journal of Visualized Experiments, 75, e2609. https://doi.org/10.3791/2609

Deussing, J. (2006). Animal models of depression. Drug Discovery Today: Disease Models, 3(4), 375-383. https://doi.org/10.1016/j. ddmod.2006.11.003 
Diehl, D., Gershon, S. (1992). The role od dopamine in mood disorders. Comprehensive Psychiatry, 33(2), 115-120. https://doi. org/10.1016/0010-440X(92)90007-D

Duncan, L., Hutchison, K., Carey, G., Craighead, W. (2009). Variation in brain-derived neurotrophic factor (BDNF) gene is associated with symptoms of depression. Journal of Affective Disorders, 115, 215-219. https://doi.org/10.1016/j.jad.2008.08.016

Egan M., Kojima, M., Callicott, J., Goldberg, T., Kolachana, B., Bertolino, A., Zaitsev, E., Gold, B., Goldman, D., Dean, M., Lu, B., Weinberger, D. (2003). The BDNF val66met polymorphism affects activity-dependent secretion of bdnf and human memory and hippocampal function. Cell, 112(2), 257-269. https://doi. org/10.1016/S0092-8674(03)00035-7

Erickson, K., Miller, D., Roecklein, K. (2012). The Aging Hippocampus: Interactions between Exercise, Depression, and BDNF. Neuroscientist, 18(1), 82-97. https://doi. org/10.1177/1073858410397054

Escorihuela, R., Fernandez-Terual, A. (1998). Modelos animales en psicopatología y psicofarmacología: del análisis experimental de la conducta a la neurogenética. Psicología Conductual, 6(1), 165-191.

Frey, B., Andreazza, A., Cereser, K., Martins, M., Valvassori, S., Reus, G., Quevedo, J., Kapezinski, F. (2006). Effects of mood stabilizers on hippocampus BDNF levels in an animal model of mania. Life Sciences, 79, 281-286. https://doi.org/10.1016/j. lfs.2006.01.002

Gómez-Restrepo, C., Bohórquez, A., Pinto Masis, D., Gil Laverde, JFA., Rondón Sepúlveda, M., Díaz Granados, N. (2004). Prevalencia de depresión y factores asociados con ella en la población colombiana. Revista Panamericana de Salud Pública, 16(6), 378-86. https://doi.org/10.1590/S1020-49892004001200003 Goodale, E. (2007). Síntomas cognitivos de la depresión. Revista de Toxicomanias, 50, 13-15. 
Hauser, T., Iannaccone, R., Walitza, S., Brandeis, D., Brem, S. (2015). Cognitive flexibility in adolescence: Neural and behavioral mechanisms of reward prediction error processing in adaptive decision-making during development. NeuroImage, 104, 347-354. https://doi.org/10.1016/j.neuroimage.2014.09.018

Healy, D. (1987). The comparative psychopathology of affective disorders in animals and humans. Journal of Psychopharmacology, 1(3), 193-210. https://doi.org/10.1177/026988118700100306 Held, S., Stanek, L., Chhatwal, J., Ressler, K. (2007). Hippocampusspecific deletion of BDNF in adult mice impairs spatial memory and extinction of aversive memories. Molecular Psychiatry, 12, 656-670. https://doi.org/10.1038/sj.mp.4001957

Ikram, H., Haleem, D. (2017). Repeated treatment with reserpine as a progressive animal model of depression. Pakistan Journal of Pharmaceutical Sciences, 30(3), 897-902.

Jiang, X., Xu, K., Hoberman, J., Tian, F., Marko, AJ., Waheed, JF., Harris, CR., Marini, AM., Enoch, MA., Lipsky, RH. (2005). BDNF variation and mood disorders: a novel functional promoter polymorphism and Val66Met are associated with anxiety but have opposing effects. Neuropsychopharmacology, 30(7), 1353-61. https://doi.org/10.1038/sj.npp.1300703

Khadrawy, Y., Sawie, H., Abdel-Salam, O., Hosny, E. (2017). Cannabis exacerbates depressive symptoms in rat model induced by reserpine. Behavioural Brain Research, 324, 41-50. https://doi. org/10.1016/j.bbr.2017.02.015

Kiss, A., Delattre, A., Pereira, S., Carolino, R., Szawka, R,. AnselmoFranci, J., Zanata S., Ferraz, AC. (2012). 17ß-estradiol replacement in young, adult and middle-aged female ovariectomized rats promotes improvement of spatial reference memory and an antidepressant effect and alters monoamines and BDNF levels in memory- and depression-related brain areas. Behavioural Brain Research, 227(1),100-8. https://doi. org/10.1016/j.bbr.2011.10.047 
Laborda, M. (2009). Modelos animales en psicopatología experimental: miedo, tolerancia a las drogas y condicionamiento. Revista de Psicología, 18(2), 81-104. https://doi.org/10.5354/0719-0581. 2009.17122

Leite, M., Perez-Acosta, A. (2001). Modelos animales en psicopatología: ¿una contribución o una ilusión? Avance en Psicología Clinica Latinoamericana, 19, 37-50.

McGorry, PD., Purcell, R., Goldstone, S., Amminger, GP. (2011). Age of onset and timing of treatment for mental and substance use disorders: implications for preventive intervention strategies and models of care. Current Opinion in Psychiatry, 24(4), 301-306. https://doi.org/10.1097/YCO.0b013e3283477a09

Mizuno, M., Yamada, K., Olariu, A., Nawa, H., Nabeshima, T. (2000). Involvement of brain-derived neurotrophic factor in spatial memory formation and maintenance in a radial arm maze test in rats. Journal of Neuroscience, 20(18), 7116-21. https://doi. org/10.1523/JNEUROSCI.20-18-07116.2000

Moussavi, S., Chatterji, S., Verdes, E., Tandon, D., Patel, V., Ustun, D. (2007). Depression, chronic diseases, and decrements in health: results from the World Health Surveys. The Lancet, 370(9590), 851-858. https://doi.org/10.1016/S0140-6736(07)61415-9

Nestler, E., Hyman, S. (2010). Animal models of neuropsychiatric disorders. Nature Neuroscience, 13(10), 1161-1169. https://doi. org/10.1038/nn.2647

OMS. (1992). CIE-10. Décima revisión de la clasificación internacional de las enfermedades. Trastornos mentales y del comportamiento. Descripción clínicas y pautas para el diagnóstico. Madrid: Ed. Méditor.

Overmier, J. (2007). La investigación básica con animales fortalece la ciencia y la práctica de la psicología. Interdisciplinaria, 24(2), 211-228.

Overstreet, D., Rezvani, A., Djouma, E., Parsian, A., Lawrence, A. (2007). Depressive-like behavior and high alcohol drinking co-occur in the FH/WJD rat but appear to be under independent 
genetic control. Neuroscience and Biobehavioral Reviews, 31(1), 103-114. https://doi.org/10.1016/j.neubiorev.2006.07.002 Rocha, TB., Zeni, CP., Caetano, SC., Kieling, C. (2013). Mood disorders in childhood and adolescence. Revista Brasileira de Psiquiatria, 35 Supl 1, S22-31. https://doi.org/10.1590/1516-4446-2013-S106 Rosso, G., Cal, K., Damián, JP., Ruiz, P., Elizondo, V., Rodriguez, H., Ferreira, A., Sotelo, JR.; Vazquez, C., Kun, A. (2010). Early phenotypical diagnoses in Trembler-J mice model. Journal of Neuroscience Methods, 190, 14-19. https://doi.org/10.1016/j. jneumeth.2010.04.010

Ruiz, P., Calliari, A., Pautassi, R. (2017). Consumo de alcohol en ratas adolescentes tratadas con reserpina y fluoxetina. Suma Psicológica, 24(1), 67-77. https://doi.org/10.1016/j.sumpsi.2016.12.002

Ruiz, P., Genovese, P., Bielli, A., Herrera, E., Pautassi, P. (2018). La reserpina aumenta la expresión de BDNF y PCNA, y disminuye la de caspasa-3, en células intersticiales (de Leydig) de rata. International Journal of Morphology, 36(3), 895-900. https://doi. org/10.4067/S0717-95022018000300895

Ruiz, P., Calliari, A., Genovese, P., Scorza, C., Pautassi, R. (2018). Amphetamine, but not methylphenidate, increases ethanol intake in adolescent male, but not in female, rats. Brain and Behaviour, e00939. https://doi.org/10.1002/brb3.939

Ruiz, P., Calliari, A., Pautassi, R. (2018). Reserpine-induced depression is associated in female, but not in male, adolescent rats with heightened, fluoxetine-sensitive, ethanol consumption. Behavioural Brain Research, 21(348), 160-170. https://doi. org/10.1016/j.bbr.2018.04.011

Sahay, A \& Hen, R. (2007). Adult hippocampal neurogenesis in depression. Nature Neuroscience, 10, 1110-1115. https://doi. org/10.1038/nn1969

Sheline, Y. I., Sanghavi, M., Mintun, M. A., \& Gado, M. H. (1999). Depression duration but not age predicts hippocampal Vol. loss in medically healthy women with recurrent major depression. 
The Journal of Neuroscience, 19(12), 5034-5043. https://doi. org/10.1523/JNEUROSCI.19-12-05034.1999

Spear, L. (2000). Modeling adolescent development and alcohol use in animals. Alcohol Research \& Health, 24(2), 115-123.

Sun, M., Alkon, D. (2005). Dual effects of bryostatin-1 on spatial memory and depression. European Journal of Pharmacology, 512(1), 43-51. https://doi.org/10.1016/j.ejphar.2005.02.028

Towbin, H., Staehelin, T., Gordon, J. (1979). Electrophoretic transfer of proteins from polyacrylamide gels to nitrocellulose sheets: procedure and some applications. Proceedings of the National Academy of Sciences of USA, 76(9), 4350-4. https://doi. org/10.1073/pnas.76.9.4350

Vaishnav, K., Nestler, E. (2011). Animal models of depression: molecular perspectives. Current Topics in Behavioral Neurosciences, 7, 121-147. https://doi.org/10.1007/7854_2010_108

Vitalis, T., Cases, O., Passemard, S., Callebert, J., Parnavelas, JG. (2007). Embryonic depletion of serotonin affects cortical development. European Journal of Neuroscience, 26(2), 331-344. https://doi.org/10.1111/j.1460-9568.2007.05661.x

Voicu, H \& Schmajuk, N. (2002). Latent learning, shortcuts and detours: a computational model. Behavioural Processes, 59(2), 67-86. https://doi.org/10.1016/S0376-6357(02)00060-8

Recibido: 29/05/2019

Revisado: 22/01/2020

Aceptado: 23/01/2020 